\title{
A promising method for the analysis of notch effect on fatigue strength: Strain energy density approach
}

\author{
SUN ChengQi ${ }^{1,2} \&$ HONG YouShi ${ }^{1,2^{*}}$ \\ ${ }^{1}$ Key Laboratory of Nonlinear Mechanics, Institute of Mechanics, Chinese Academy of Sciences, Beijing 100190, China; \\ ${ }^{2}$ University of Chinese Academy of Sciences, Beijing 100049, China
}

Received August 25, 2016; accepted September 5, 2016; published online September 13, 2016

In recent years, the approach of strain energy density (SED) has received special attention in the analysis of notch effect on fatigue strength of notched components, e.g. ref. [1-3]. One of the fundamental contributions to SED-based approaches was by Sih [4,5] who proposed the SED factor $S$, which was defined as the product of the SED and a critical distance from the point of singularity. Thus, the failure is controlled by the critical value $S_{\mathrm{c}}$.

The SED approach is based on the idea that under tensile stresses, failure occurs when the strain energy density $\bar{W}$ equals to the critical value $W_{\mathrm{c}}$, which needs a precise definition of the related control volume. If the material is ideally brittle, the value of $W_{\mathrm{c}}$ can be evaluated by $0.5 \sigma_{\mathrm{t}}^{2} / E$. Commonly, unnotched specimens exhibit a non-linear behavior whereas the behavior of notched specimens remains linear. Therefore, the stress $\sigma_{\mathrm{t}}$ should be substituted by the maximum normal stress existing at the edge at the moment preceding the cracking [6]. In particular, for plane problems, the control volume becomes a circle or a circular sector with a radius $R_{0}$ in the case of cracks or sharp V-notches with mode I or mixed I + II mode loading [6], as shown in Figure 1(a) and (b). Further, for the case of crack problems under plane strain conditions, the expression for $R_{0}$ has been derived $[6,7]$ :

$$
R_{0}=\frac{(1+v)(5-8 v)}{4 \pi}\left(\frac{K_{\mathrm{IC}}}{\sigma_{\mathrm{t}}}\right)^{2} .
$$

If the critical value of the notch stress intensity factor is determined via the specimens with $2 \alpha \neq 0$, the critical radi-

\footnotetext{
*Corresponding author (email: hongys@imech.ac.cn)
}

us is calculated by

$$
R_{0}=\left(\frac{I_{1} \times K_{\mathrm{IC}}^{2}}{4 \lambda_{1}(\pi-\alpha) E W_{\mathrm{c}}}\right)^{1 /\left(2-2 \lambda_{1}\right)},
$$

where $I_{1}$ is a parameter, $\lambda_{1}$ is Williams' eigenvalue and $K_{\mathrm{IC}}$ is the fracture toughness at $2 \alpha=0$.

For the case of blunt notch, the cross section area is treated as a crescent shape, with $R_{0}$ being its maximum width as measured along the notch bisector line, as shown in Figure 1(c). Under mixed-mode loading, the control area is no longer centered with respect to the notch bisector. It is centered on the point where the maximum principal stress reaches its maximum value [7].

Unlike the calculation of notch stress intensity factor, the mean value of SED on the control volume has the advantage that it can be determined with high accuracy by using coarse meshes in finite element calculations [8,9], which has been widely used for sharp, zero radius, V-notches, blunt U- and V-notches under mode I loading, and also applied to welded joints and notched specimens under multi-axial loading. In this aspect, Berto and Lazzarin [2,6,8] have made important contributions to the development and application of SED approach for notched components and welded joints. A specific work by Lazzarin and Zambardi [10] used the local SED to predict the static and fatigue behavior of the components with sharp V-shaped notches. Recently, Berto and Lazzarin [9] first applied the SED approach to welded joints and notched plates of 7075-T651 aluminum alloy widely for aerospace applications. As an example, Figure 1(d) shows the fatigue data of the notched specimens made of C40 carbon steel (normalized state), which is subjected to combined tension and torsion loading, in terms of the total SED at the 
(a)

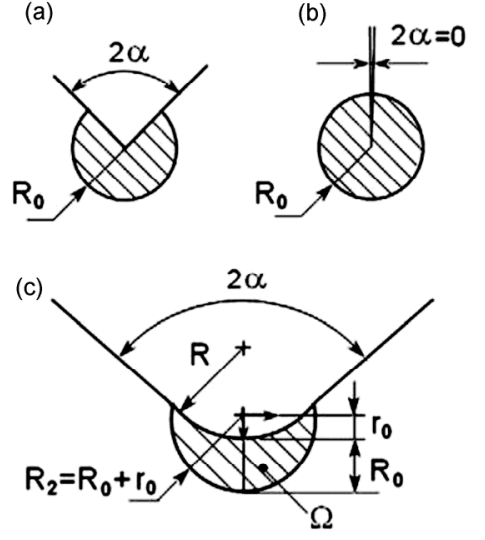

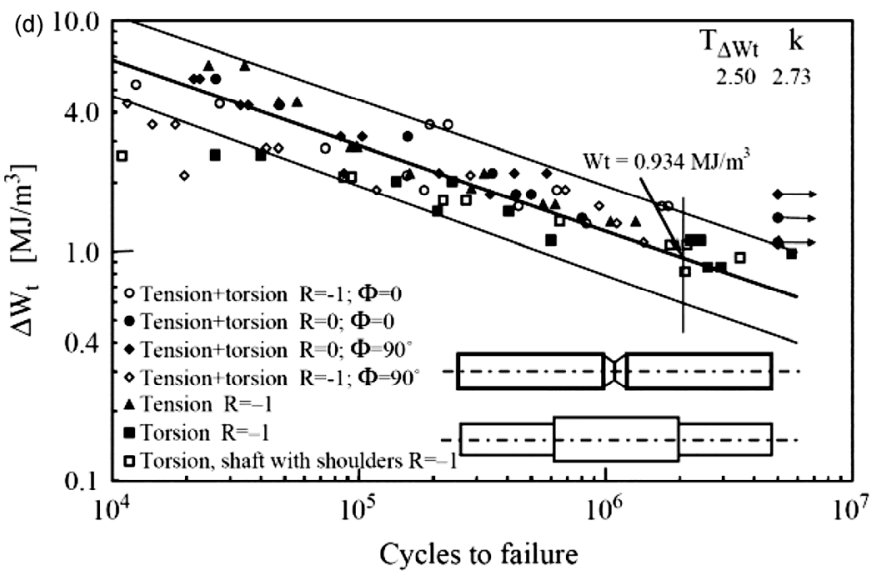

Figure 1 Critical volume (area) for sharp V-notch (a), crack (b) and blunt V-notch (c) under mode I loading, with $r_{0}=R \times(\pi-2 \alpha) /(2 \pi-2 \alpha)[6]$, and (d) fatigue strength data of notched specimens in terms of total strain energy density at notch tip [11].

notch tip with $10 \%-90 \%$ scatter band of the fatigue curve [11].

As a method for fatigue strength assessment of notch effect, the SED approach has been successfully used in notched and welded specimens subjected to both uniaxial and multi-axial fatigue loadings. Due to its unique nature, it is capable of taking into account the actual degree of multiaxiality for the stress field of fatigue process zone [12]. However, the failure criterion for large specimen (high volume to surface ratio) may fail to be directly extend to small scale specimens where the local inhomogeneities play a substantial role in the fatigue crack initiation and propagation $[1,6]$. Therefore, it is the next step to develop a multiscaling or segmentation scheme able to incorporating the specimen size effect.

1 Fischer C, Fricke W, Rizzo C M. Review of the fatigue strength of welded joints based on the notch stress intensity factor and SED approaches. Int J Fatigue, 2016, 84: 59-66

2 Berto F, Campagnolo A, Lazzarin P. Fatigue strength of severely notched specimens made of Ti-6Al-4V under multiaxial loading. Fatigue Fract Eng Mater Struct, 2015, 38: 503-517
3 Tang X S. Scatter of fatigue data owing to material microscopic effects. Sci China Phys Mech Astron, 2014, 57: 90-97

4 Sih G C. Strain-energy-density factor applied to mixed mode crack problems. Int J Fracture, 1974, 10: 305-321

5 Sih G C. From monoscale to multiscale modeling of fatigue crack growth: Stress and energy density factor. Sci China Phys Mech Astron, 2014, 57: 39-50

6 Berto F, Lazzarin P. A review of the volume-based strain energy density approach applied to V-notches and welded structures. Theor Appl Fract Mech, 2009, 52: 183-194

7 Yosibash Z, Bussiba A, Gilad I. Failure criteria for brittle elastic materials. Int J Fracture, 2004, 125: 307-333

8 Lazzarin P, Berto F. Control volumes and strain energy density under small and large scale yielding due to tension and torsion loading. Fatigue Fract Eng Mater Struct, 2008, 31: 95-107

9 Berto F, Lazzarin P. Fatigue strength of A17075 notched plates based on the local SED averaged over a control volume. Sci China Phys Mech Astron, 2014, 57: 30-38

10 Lazzarin P, Zambardi R. A finite-volume-energy based approach to predict the static and the fatigue behaviour of components with sharp V-shaped notches. Int J Fracture, 2001, 112: 275-298

11 Atzori B, Berto F, Lazzarin P, et al. Multi-axial fatigue behaviour of a severely notched carbon steel. Int J Fatigue, 2006, 28: 485-493

12 Susmel L. The theory of critical distances: A review of its applications in fatigue. Eng Fract Mech, 2008, 75: 1706-1724 